\title{
EFECTO DE LA ORCIPRENALINA Y DEL Th-1165-a SOBRE LA CONTRACTILIDAD DEL UTERO MENSTRUAL HUMANO**
}

\author{
Dr. Rodrigo Cifuentes B., M.D., Ph.D.泳澡

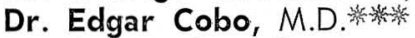

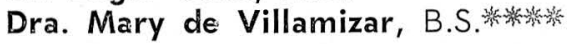

\section{Resumen}

Se estudia el efecto de los fármacos estimulantes de los adrenoceptores beta administrados por vía sublingual, sobre la contractilidad uterina durante la menstruación. Los fármacos estudiados fueron el Th-1165-a (Partusisten) y la Orciprenalina (Alupent ).

Se registró la contractilidad uterina mediante un catéter abierto de polietileno con esponja terminal. Se analizaron cuantitativamente los registros antes y después de la administración sublingual de los fármacos mencionados. También se analizó el efecto de ellos sobre la osmolaridad urinaria mediante recolección continua de la orina.

Con ambos fármacos se objetivó una disminución estadísticamente significativa de la intensidad, tono, frecuencia y actividad uterina en todas las pacientes. En las pacientes que presentaban dismenorrea esencial, concomitantemente con la disminución de la contractilidad uterina hubo abolición subjetiva del dolor pélvico.

La Orciprenalina produjo además, disminución significativa de la osmolaridad urinaria.
Se concluye sugiriendo la posible utilidad terapéutica de estos fármacos en la clínica ginecológica en pacientes con dismenorrea esencial.

\section{Introducción}

Ha sido demostrada por diversos autores $(6,8,11)$ la existencia en el útero humano de adrenoceptores alfa y beta. Es así, como la estimulación farmacológica de los receptores beta causa inhibición de la actividad contráctil del útero humano grávido (2, $5,7,10)$ lo cual tiene amplia aplicación clínica en el manejo de algunas entidades que requieren inhibición de la contractilidad uterina, como son amenaza de parto prematuro (5) o el sufrimiento fetal agudo intrapar. to $(3)$.

* Trabajo auspiciado por la Organización Mundial de la Salud.

* El Dr. Cifuentes es Investigador asociado, Unidad de Fisiología de la Reproducción, Departamento de Obstetricia y Ginecología, Universidad del Valle, Cali, Colombia.

$* * *$ El Dr. Cobo es Profesor-Jefe del Departamento de Obstetricia y Ginecología, Universidad del Valle, Cali, Colombia.

**** La Dra. de Villamizar es Técnica de Laboratorio, Unidad de Fisiología de la Reproducción, Departamento de Obstetricia y Ginecología, Universidad del Valle, Cali, Colombia. 
El objetivo del presente trabajo fue el de estudiar el efecto de dos fármacos estimulantes de los adrenoceptores beta, administrados por vía sublingual, sobre la contractilidad uterina durante los primeros días del ciclo menstrual. Los fármacos estudiados fueron el Th-1165-a (Partusis$\operatorname{ten}^{R}$ ) y la Orciprenalina (Alupent ${ }^{R}$ ) cuyos efectos sobre el útero grávido son bastante conocidos, especialmente la Orciprenalina (potente úteroinhibidor) (9). Sin embargo, se desconocían los efectos sobre la contractilidad uterina que pudieran tener estos fármacos al ser administrados por vía sublingual durante la menstruación.

\section{Material y Métodos}

Se estudiaron 40 pacientes normales, en edad fértil, con ciclos menstruales regulares y de una duración que oscilaba entre 26 y 30 días. Además, no padecían de patología genital - cardiovascular detectable. De estas 40 se seleccionaron 20 voluntarias a quienes se les efectuó registro de contractilidad uterina durante los días 1 a 4 del ciclo menstrual.

Los fármacos, vía y dosis utilizados fueron:

Th-1165-a (Partusisten). Es un compuesto sintético perteneciente al grupo de las resorciletanolaminas, al igual que la Orciprenalina, del cual deriva por sustitución de uno de los hidrógenos de un carbono extremo del radical isopropílico por un radical fenólico, con el hidroxilo en posición para. Esta modificación química parece que incrementa la afinidad del producto por los adrenoceptores beta. En este trabajo se utilizó el Th1165 -a en tabletas de $5 \mathrm{mg}$. Después de media hora de registro continuo de contractilidad uterina, se administró el fármaco a 10 pacientes a la dosis de $10 \mathrm{mg}$. vía sublingual. Esta dosis se repitió media hora después. El registro se continuó por 3 horas más.

Orciprenalina (Alupent). Es una sustancia sintética muy conocida, de gran afinidad por los adrenoceptores beta con muy poca acción sobre los adrenoceptores alfa. La Orciprenalina se administró a dosis de $20 \mathrm{mg}$. por vía sublingual, previo registro estable de contractilidad uterina espontánea durante media hora. La dosis de Orciprenalina se repitió media hora más tarde. El registro continuó durante $2 \frac{1 / 2}{2}$ a 3 horas.

\section{Métodos de registro}

En todos los estudios en los cuales se registró continuamente la contractilidad uterina, se empleó el método de Bengtsson (1). Consiste en registrar la presión intrauterina mediante un catéter de polietileno (P. E. 160 Clay Adams) en cuyo extremo terminal abierto se coloca una pequeña esponja. El catéter se coloca por vía vaginal a través del cérvix en la cavidad uterina. El otro extremo del catéter se concta a un transductor eléctrico de presiones y éste a un polígrafo multicanal (1).

Además, se tomó una muestra sanguínea de vena antecubital, al inicio de la experiencia, para medidas de osmolaridad sérica y se colocó una sonda de Folley en la vejiga durante el tiempo que duró el registro para recolección continua de orina y medidas de osmolaridad.

Análisis de registros. Se consideraron como contracciones uterinas a las inflexiones del registro iguales 0 superiores a $5 \mathrm{~mm}$ de $\mathrm{Hg}$. En cada contracción se midió para períodos de 30 minutos, la intensidad (en $\mathrm{mmHg}$ ), el tono (en $\mathrm{mmHg}$ ), la frecuencia (calculada mediante el inter- 


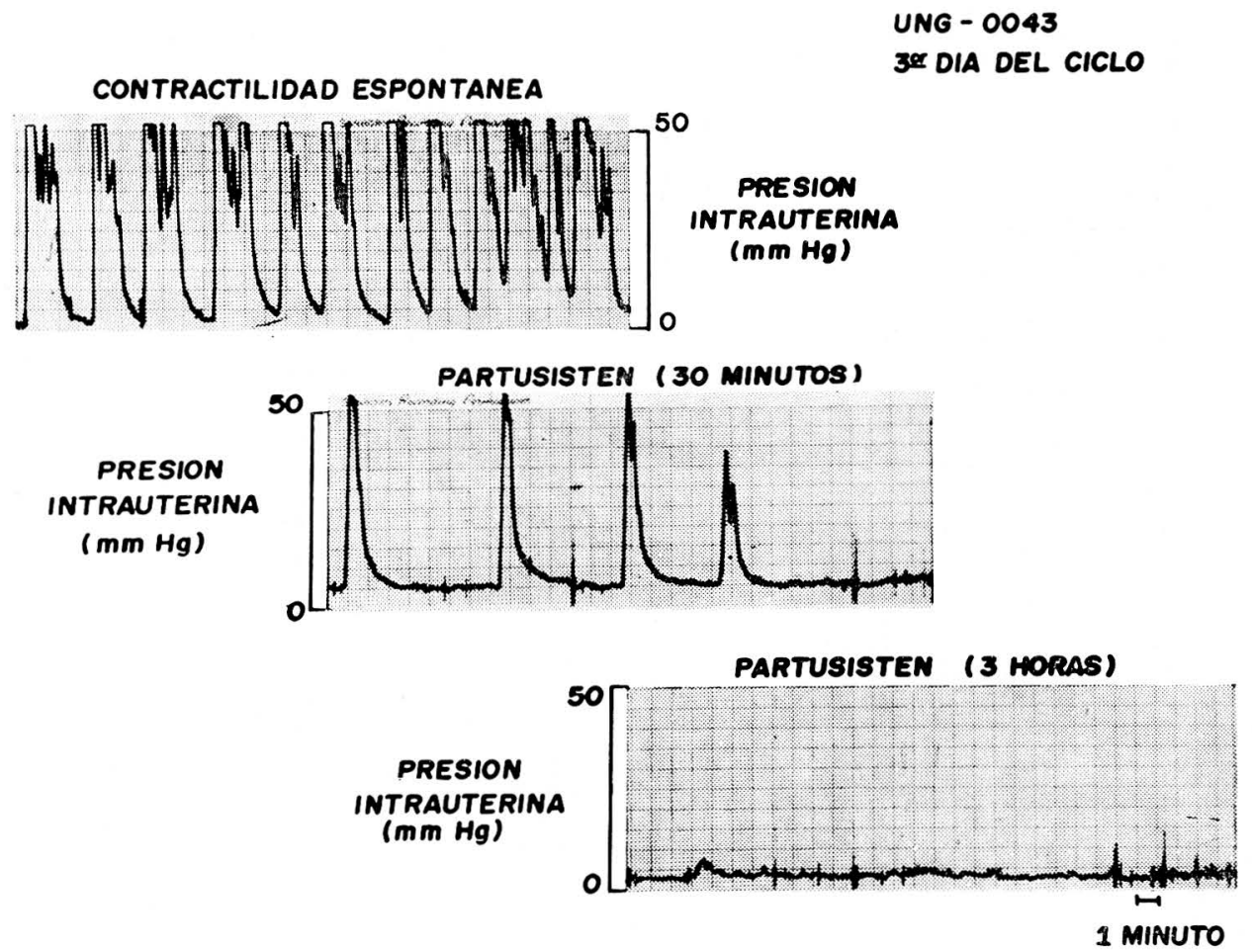

FIGURA 1 - Registro de presión intrauterina en una mujer durante el tercer día del ciclo menstrual. Nótese, en la parte superior la contractilidad uterina espontánea, en la parte media, la contractilidad uterina 30 minutos después de administrar Th-1165-a (Partusisten) sublingual. En la parte inferior, el mismo registro 3 horas después donde aún persiste la abolición casi completa de la contractilidad uterina. 


\section{UTERO NO GRAVIDO}

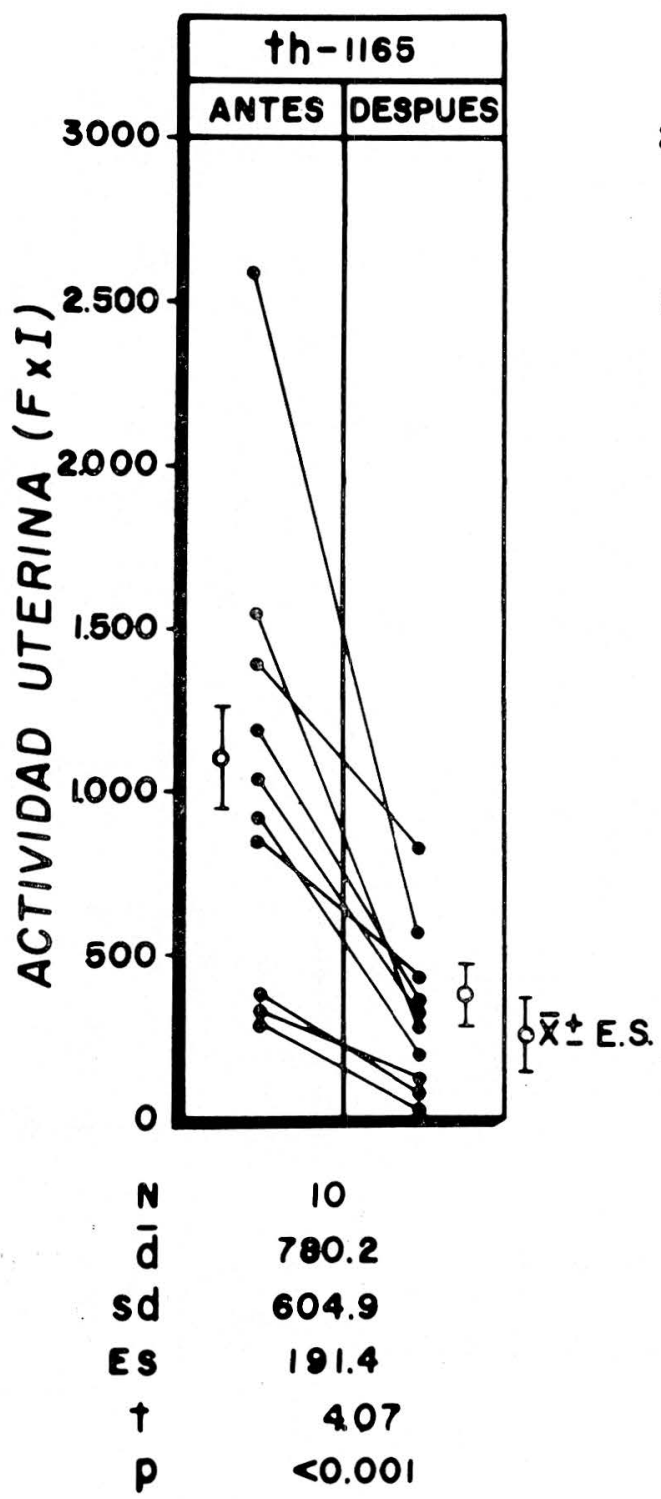

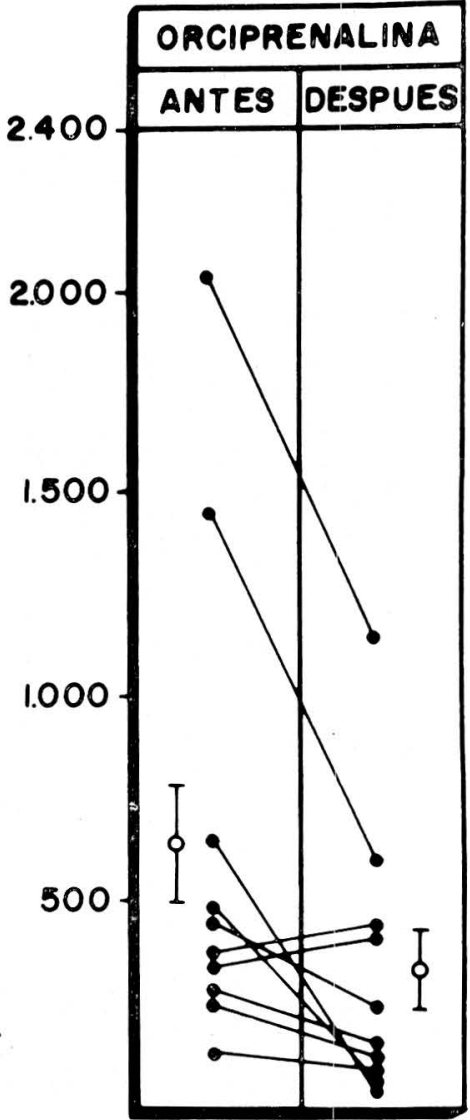

10

304.5

348.1

110.2

2.76

$<0.02$

FIGURA 2 - Actividad uterina (frecuencia en 30 minutos por intensidad en $\mathrm{mmHg}$ ) antes y después de la administración sublingual de Th-1165-a (Partusisten) y Orciprenalina. Con ambos fármacos se obtiene inhibición de la contractilidad ứerina estadísticamente significativa. 
valo temporal con la contracción precedente) y la actividad uterina (producto de la intensidad por la frecuencia). Con los datos obtenidos para cada uno de los parámetros, se realizaron análisis estadísticos mediante el test de Student (" $t$ ") para muestras dependientes.

\section{Resultados}

Th-1165-a. Durante la administración de este fármaco se objetivó una disminución de todos los parámetros de la contractilidad uterina respecto de los valores basales (Figs. 1 y 2 ). Aunque el efecto útero-inhibidor del fármaco es bastante evidente, se realizó el análisis estadístico mediante el test de " $\mathrm{t}$ " ya mencionado y las diferencias fueron altamente significativas (Tabla 1).

La disminución de la contractilidad uterina producida por el Th1165-a fue de larga duración. En la mayoría de los casos, tres horas después de administrado el fármaco, la contractilidad uterina permanecía abolida (Fig. 1). Igualmente, el Th1165-a produjo disminución de la Osmolaridad Urinaria, aunque ésta no fue estadísticamente significativa (Fig. $3)$.

En cuanto a los efectos cardiovasculares, se produjo un ligero incremento de la frecuencia cardíaca basal (de 10 a 15 lat/min.) con disminución de la presión arterial diastólica (en $10 \mathrm{mmHg}$ ) y aumento de la presión arterial diferencial (en 10 $\mathrm{mmHg}$ ).

Orciprenalina. La administración de esta droga produjo una inhibición casi inmediata de la actividad y el tono uterinos, la cual persistió más de 2 horas (Figs. 4 y 5 ). En algunas pacientes dismenorreicas en quienes también se utilizó esta droga, la inhibición de la contractilidad uterina

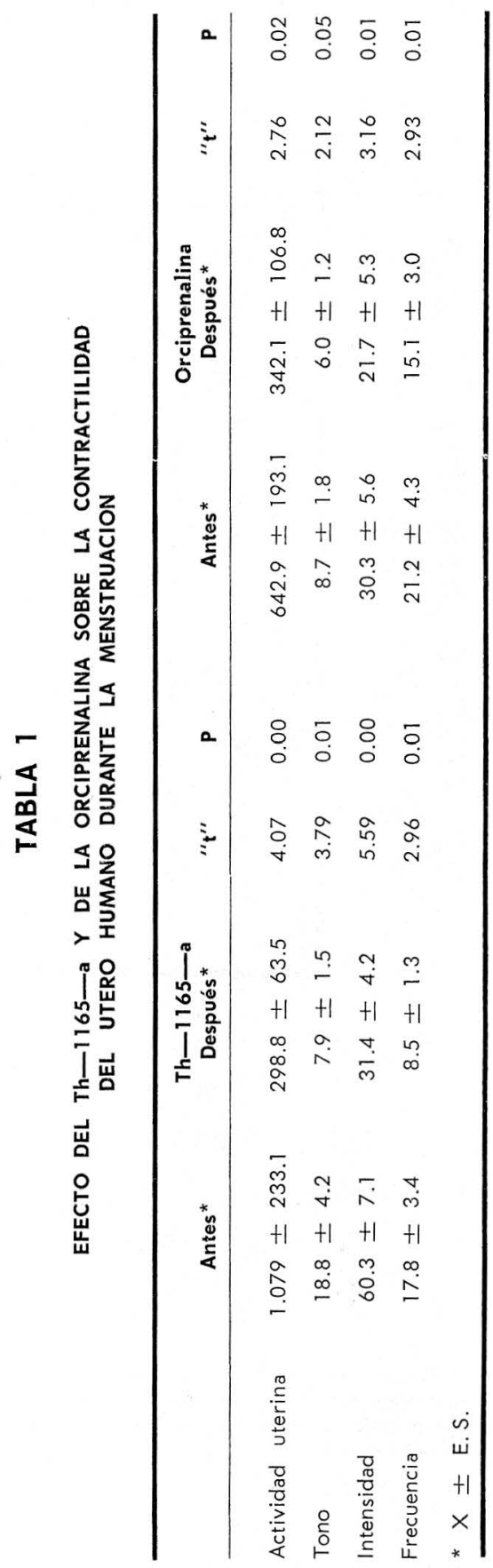




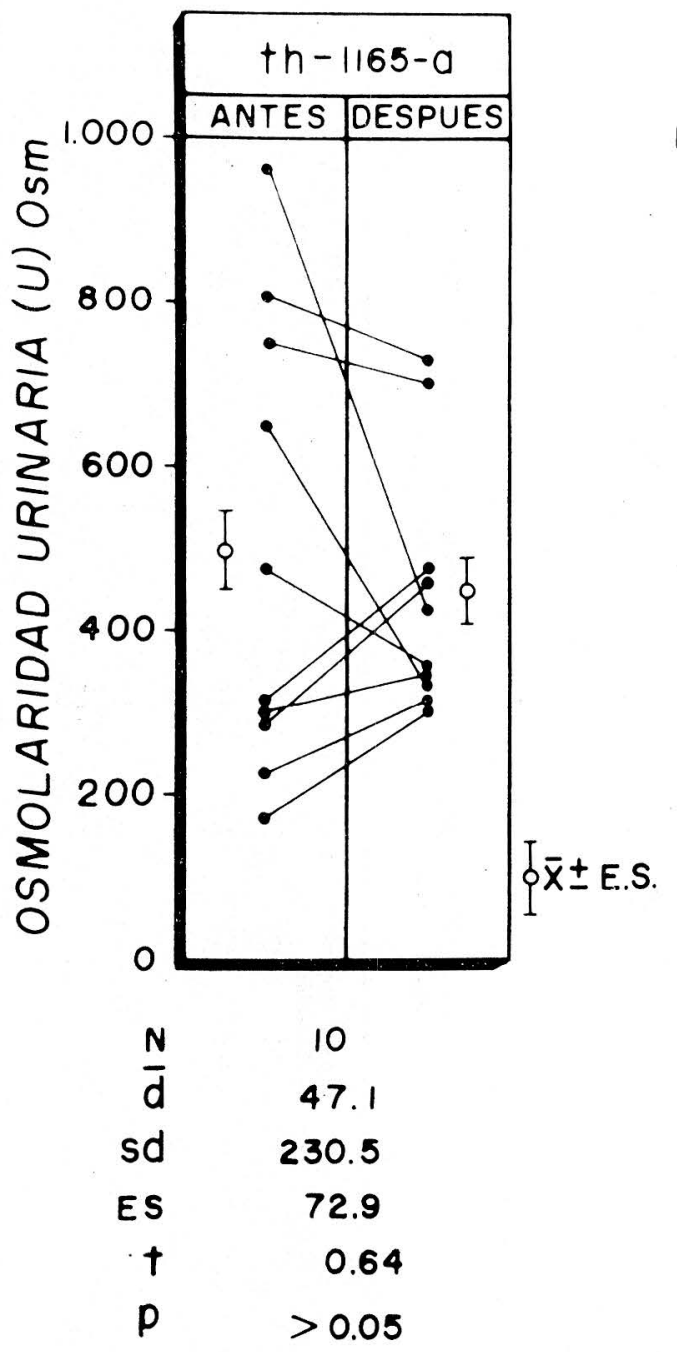

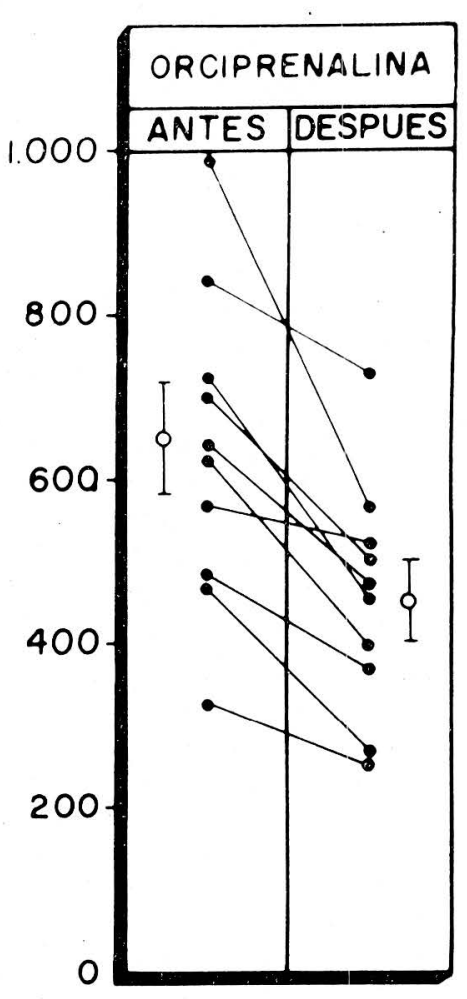

10

155.7

122.2

38.7

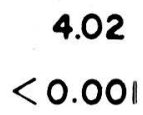

FIGURA 3 - Efecto del Th-1165-a (Partusisten) y la Orciprenalina (Alupent) administrados por vía sublingual, sobre la osmolaridad urinaria. Con la orciprenalina se observa una disminución de la osmolaridad estadísticamente significativa, no así con el Th-1165-a. 
Vol. XXVII
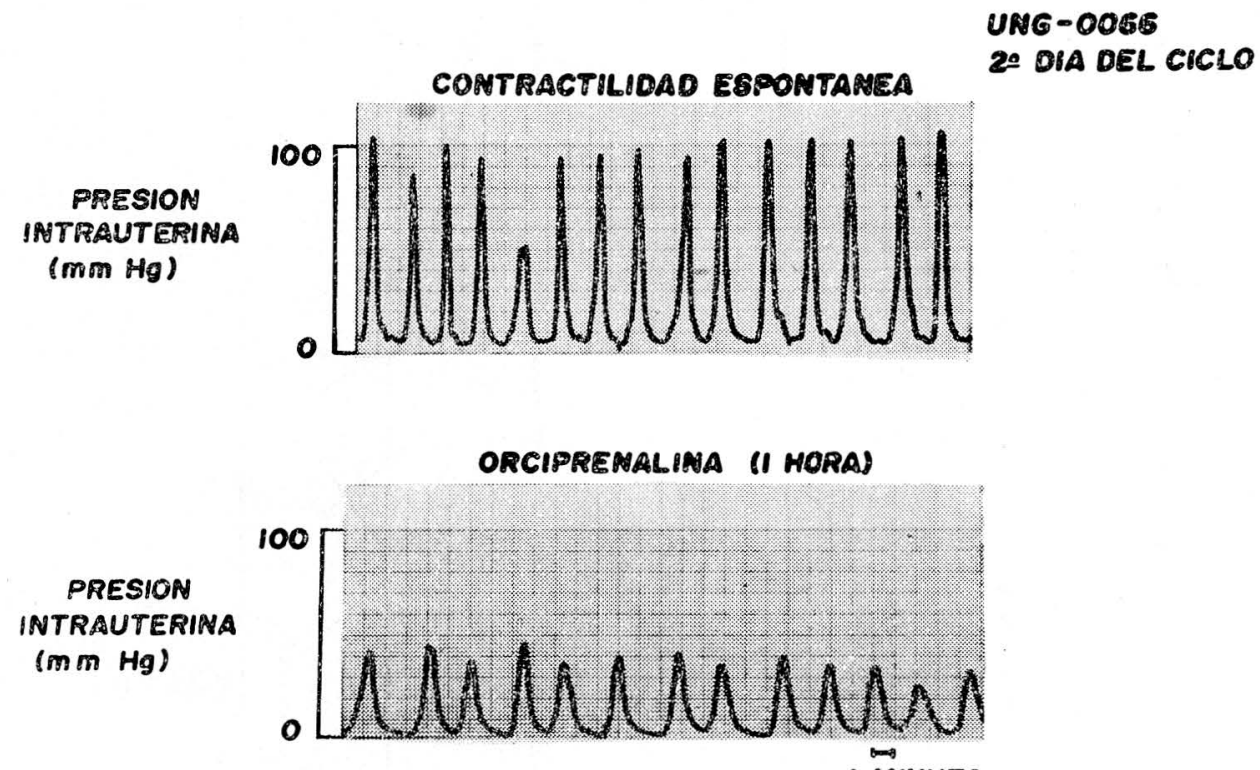

1 AMUTO

FIGURA 4 - Fragmento de un registro de presión intrauterina realizado en una mujer normal durante el segundo día del ciclo menstrual. Se puede observar el claro efecto útero-inhibidor de la orciprenalina (Alupent) administrada por vía sublingual. Los efectos cardiovasculares fueron mínimos y bien tolerados. 


\section{UTERO NO GRAVIDO}
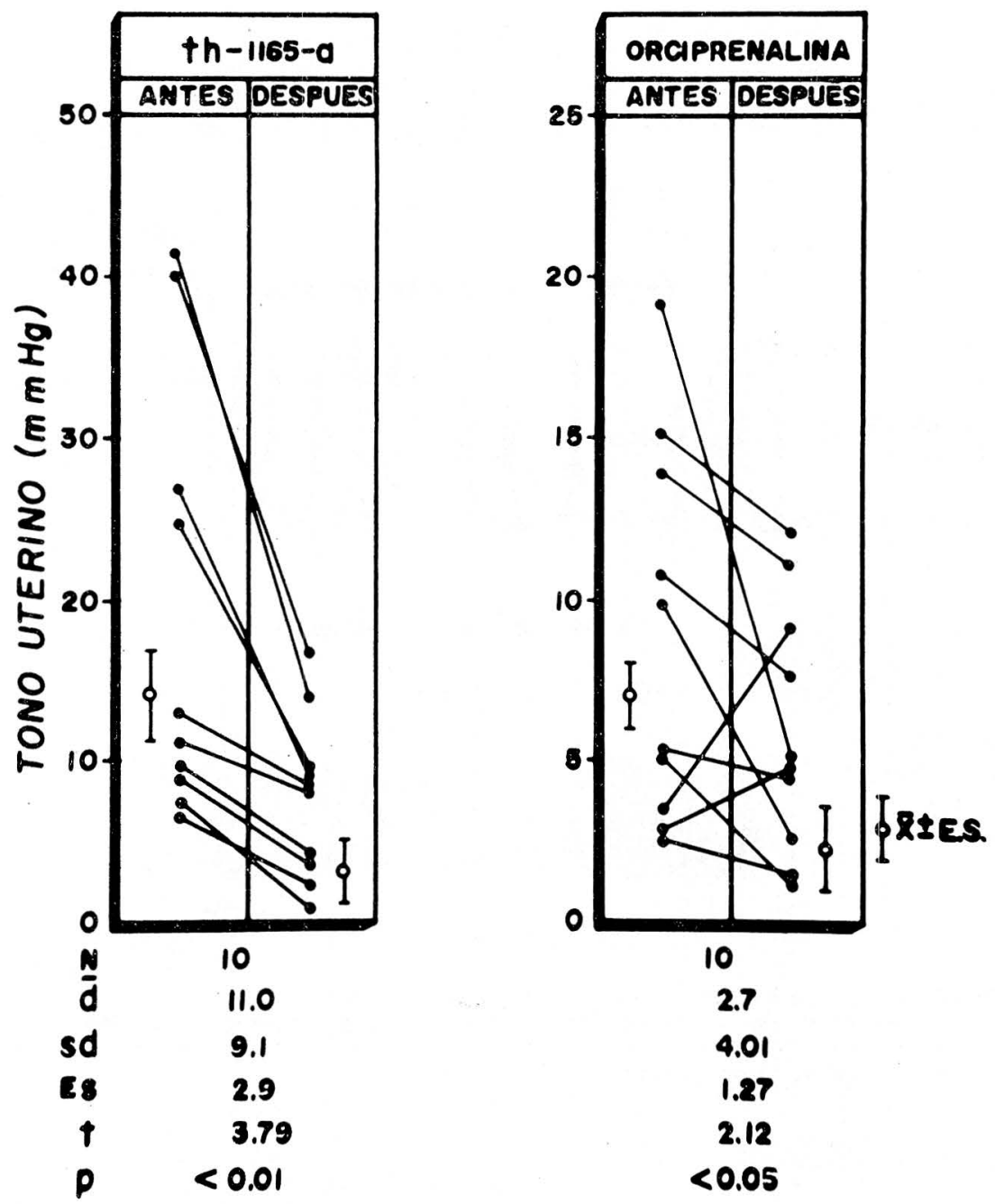

FIGURA 5 - Efecto del Th-1165-a (Partusisten) y la Orciprenalina (Alupent) administrados por vía sublingual, sobre el tono uterino (en $\mathrm{mmHg}$ ) durante la menstruación. Con ambos fármacos se observa una disminución intensa, estadísticamente significativa, del parámetro mencionado. 
coincidió con la abolición completa del dolor pélvico.

La Orciprenalina produjo además disminución de la osmolaridad urinaria, la cual, analizada mediante el test ya mencionado, fue estadísticamente significativa (Fig. 3). Igualmente, se observó aumento de la frecuencia cardíaca basal (de 15 a 20 lat $/ \mathrm{min}$.) disminución de la presión arterial diastólica (15 a $20 \mathrm{mmHg}$ ) y aumento de la presión arterial diferencial (15 a $20 \mathrm{mmHg}$ ).

Estos efectos cardiovasculares fueron bien tolerados por las pacientes y su duración mucho menor que los efectos uterinos.

\section{Discusión y Comentarios}

El intenso efecto útero-inhibidor del Th-1165-a coincide con publicaciones previas de experimentos efectuados con igual metodología y aplicando el fármaco por vía intravenosa (4). Además, este fármaco por vía sublingual y a las dosis empleadas en la presente investigación produjo una estimulación de los adrenoceptores beta suficiente para inhibir la contractilidad uterina con muy pocos efectos cardiovasculares.

Respecto de la Orciprenalina, no existe experiencia previa sobre su efecto en útero humano no grávido in vivo, durante la menstruación y por vía sublingual. Nuestros resultados coinciden con lo informado por otros autores en útero humano grávido $(2,5,9)$.

Es de destacar sin embargo, la acción inhibidora de la contractilidad uterina de larga duración y con efectos cardiovasculares bien tolerados por las pacientes. Además, en las ex- periencias en que se utilizó este fármaco por vía sublingual en mujeres con dismenorrea esencial, concomitantemente con la disminución de las contracciones uterinas (demostrada objetivamente) existió una abolición del dolor pélvico.

La larga acción útero-inhibidora de estos fármacos cuando se administran por vía sublingual puede interpretarse como debida a un catabolismo más lento por la catecol-orto-metil-transferasa (CONT), ya que se obvia el paso directo de los beta-adrenérgicos por el hígado (vía vena porta) donde se pueden inactivar por la enzima mencionada.

Esta persistencia de los efectos útero-inhibidores del Th-1165-a y de la Orciprenalina, así como el efecto diurético de esta última, demostrado por la disminución significativa en la osmolaridad urinaria, pueden ser de utilidad en la clínica ginecológica para el tratamiento de las dismenorreas esenciales, entidad esta para la cual se carece hasta la fecha, de una terapia efectiva.

\section{Summary}

The effects of the beta-adrenoreceptors by sublingual route over the uterine contraction during menstruation are studied. The drugs tested were $\mathrm{TH}-1165$ a (partusisten) and orciprenalina (alupent).

Both drugs reduced greatly the uterine contractions in all the patients. In essential dismenorrhea besides the disminution of the contractility subjective pain desappeared.

Partusisten and alupent are recomended against essential dismenorrhea as conclusion of this paper. 


\section{AGRADECIMIENTO :}

Al Laboratorio Boehringer Ingelheim, S. A. por su colaboración con la donación de los fármacos Partusisten y Alupent.

\section{BIBLIOGRAFIA}

1 BENGTSSON, LARS Ph.: The sponge-tippedcatheter. A modification of the open end catheter for recording of myometrial activity in vivo. J. Reprod. Fert. 16 (1): 115, 1968.

2 BRUGGER-AUBAN, A. J.: Acción espasmolítica de la Orciprenalina y mecanismo receptor adrenérgico del útero humano. Simposio sobre drogas útero inhibidoras. Punta del Este, Uruguay: 14, 1970.

3 CALDEYRO-BARCIA, R., MAGAÑA, J. M., CASTILLO, J. B., POSEIRO, J. J., MENDEZ BAUER, C., POSE, S. V., ESCARCENA, L., CASACUBERTA, C., BUSTOS, J. R., GIUSSI, G.: Nuevo enfoque para el tratamiento del sufrimiento fetal agudo Intra-parto. Arch. Ginec. Obstet. 24: 15, 1965.

4 CIFUENTES, R.: Contractilidad del útero humano no grávido en 4 fases del ciclo menstrual. Acción de fármacos estimulantes de los adrenoceptores. Tesis para el doctorado en Ciencias Biológicas, Universidad del Salvador, Buenos Aires, Argentina. 36, 1974.

5 COBO, E., KAFURY, S.: Inhibición de la contractilidad del útero en la amenaza de parto prematuro mediante el uso de Orciprenalina (Alupent). Informe preliminar. Rev. Col. Obstet. y Ginec. 21: 111, 1970.

6 MILLER, J. W.: Adrenergic receptor in the myometrium. Ann. N.Y., Acad. Sci. 139 (3): $788,1967$.

7 MONLECN, F. J., BRUGGER, A. J.: Efectos de la administración de Orciprenalina sobre la contractilidad uterina y sobre el binomio feto-materno. Med. Klin. (Ed. esp.), $10(101): 85,1970$.

8 OWMAN, C., ROSENGREN, E., SJOBERG: Adrenergic innervation of the human female reproductive organs. A histochemical and chemical investigation. Obstetrics and Gynecology, 30: 763, 1967.

9 POSEIRO, J. J., GUEVARA-RUBIO, E., MAGAÑA, J. M., CALDEYRO-BARCIA, R.: ACción de la Orciprenalina (Alupent) sobre la contractilidad del útero humano grávido, el sistema cardiovascular materno y la frecuencia cardíaca fetal. Arch. Ginec. Obstet. 23: 99, 1968

10 SCHWARCZ, R. L.: Investigación de la acción del clorhidrato de etiladrianol sobre la contractilidad uterina en la mujer embarazada. Tesis de doctorado, Facultad de Medicina. Univ. Nal. de Buenos Aires, Argentina, 1969.

11 THEOBALD, G. W.: Nervous control of uterine activity. Clin. Obstet. Gynec. 11: 15, 1968. 